\title{
Spatial structure of an incompressible Quantum Hall strip
}

\author{
Ivan A. Larkin†and L. S. Levitov $\ddagger$ \\ $\dagger$ Department of Physics and Astronomy, University of Sheffield, Sheffield S3 7RH, UK \\ $\ddagger$ Center for Materials Science $\&$ Engineering, Physics Department, MIT, Cambridge, MA-02139
}

Abstract

The incompressible Quantum Hall strip is sensitive to charging of localized states in the cyclotron gap. We study the effect of localized states by a density functional approach and find electron density and the strip width as a function of the density of states in the gap. Another important effect is electron exchange. By using a model density functional which accounts for negative compressibility of the $\mathrm{QH}$ state, we find electron density around the strip. At large exchange, the density profile becomes nonmonotonic, indicating formation of a 1D Wigner crystal at the strip edge. Both effects, localized states and exchange, lead to a substantial increase of the strip width.

\section{Introduction}

Theory of the QHE predicts that near integer filling the system divides into compressible regions separated by incompressible strips [1]. Potential distribution within a QHE sample was recently imaged by using capacitance probes [2,3], atomic force microscope [4] and single electron transistor [5]. High resolution images of the incompressible strip [2,3] give the strip width several times larger than the theoretical prediction [1]. To bridge between theory and experiment one has to extend the analysis [1] to include

- the effect of disorder producing finite density of states in the cyclotron gap;

- electron exchange correlations which affect compressibility of the QHE state.

Below we study these effects using a Density Functional approach, taking special care of the effect of a large dielectric constant $\left(\epsilon_{\mathrm{GaAs}}=12.1\right)$. Because of relatively small depth of the 2DEG beneath the semiconductor surface, the interparticle interaction within the 2DEG is affected by image charges. This changes electrostatics of the strip, and modifies potential induced on the exposed surface.

Finite density of states in the QHE gap gives rise to a finite screening length. For an incompressible strip of width exceeding this screening length, we find a large departure from the theory [1], in agreement with [8]. The results compare well with the experiment [3].

The effect of electron exchange is important in determining the structure of compressible regions adjacent to the strip. Exchange correlation gives rise to negative compressibility [7] of the 2DEG. We consider negative compressibility by using a model density functional, and show that it strongly alters the distribution of electric charge, even to the extent that the potential and the charge density profiles can become nonmonotonic.

\section{The effect of finite density of states in the cyclotron gap}

Incompressible strips are formed in the regions of nonuniform 2DEG density, at nearly integer filling, created either by perturbing the exposed surface by an STM probe [2] or by gating the 2DEG [3]. The strips are aligned normal to the average 2DEG density gradient. Charge distribution around the strip is controlled by electrostatics $[1,6]$.

Density $n(r)$ in the 2DEG buried at a distance $d$ beneath semiconductor surface can be found by minimizing a density functional: 


$$
-U_{\text {ext }}(r)=\int V\left(r-r^{\prime}\right) n\left(r^{\prime}\right) d^{2} r^{\prime}+\mu(n), \quad V(r)=\frac{e^{2}}{\epsilon|r|}+\frac{(\epsilon-1) e^{2}}{\epsilon(\epsilon+1) \sqrt{r^{2}+4 d^{2}}}
$$

Here $U_{\text {ext }}(r)$ is external potential due to donors or gates, the Hartree interaction $V\left(r-r^{\prime}\right)$ takes into account image charges, and the chemical potential $\mu$ includes various non-Hartree contributions: finite density of states, QHE gap, exchange effects, etc.

Since the scale of the observed structures $[2,3]$ is always larger than the 2DEG depth $d$, we replace the Hartree interaction in (11) by $V(r)=2 e^{2} /((\epsilon+1)|r|)$, assuming $|r| \gg d$.

In this section we consider a free electron model for $\mu(n)$ which includes degenerate (i.e., infinitely compressible) Landau level states and localized states in the QH gap:

$$
d n / d \mu=\left(n_{\mathrm{LL}}-n_{\mathrm{gap}}\right) \sum_{m>0} \delta\left(\mu-m \hbar \omega_{c}\right)+n_{\mathrm{gap}} / \hbar \omega_{c}, \quad n_{\mathrm{LL}}=e B / h c .
$$

In the simplest model Eq.(2) the density of localized states is constant.

Below we focus on the $m=1$ QHE plateau. To introduce the 2DEG density gradient into the problem, we express $U_{\text {ext }}$ in terms of fictitious positive charge density within the 2DEG plane:

$$
U_{\mathrm{ext}}(r)=\int V\left(r-r^{\prime}\right) n_{\mathrm{eff}}\left(r^{\prime}\right) d^{2} r^{\prime}, \quad n_{\mathrm{eff}}(r)=-n_{\mathrm{LL}}-\vec{r} \cdot \vec{\nabla} n
$$

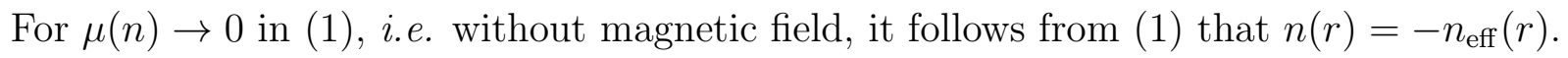

Now, we nondimensionalize the problem by choosing

$$
w_{0}=\left((\epsilon+1) \hbar \omega_{c} / 2 e^{2}|\nabla n|\right)^{1 / 2} \quad \text { and } \quad n_{0}=\left((\epsilon+1) \hbar \omega_{c}|\nabla n| / 2 e^{2}\right)^{1 / 2}
$$

as the length and density units. Then the only remaining dimensionless parameter is the ratio of the fully incompressible strip width $w_{0}$ to the screening radius $r_{s}$ corresponding to the density of states $n_{\text {gap }} / \hbar \omega_{c}$ in the gap. Up to a factor of $2 \pi$, this ratio is $\gamma=n_{\text {gap }} / n_{0}=$ $n_{\text {gap }}\left(2 e^{2} /(\epsilon+1) \hbar \omega_{c}|\nabla n|\right)^{1 / 2}$. The nondimensionalized problem reads:

$$
\int \frac{\left(x^{\prime}-\delta n\left(r^{\prime}\right)\right) d^{2} r^{\prime}}{\left|r-r^{\prime}\right|}=\int_{0}^{\delta n(r)} F_{\gamma}(u) d u
$$

where $\delta n(r)=n(r)-n_{\mathrm{LL}}$, and $F_{\gamma}(u)=\gamma^{-1}$ for $|u|<\gamma / 2$, and 0 otherwise. Here the coordinate system is such that the $x$ axis is normal to the strip, and the $y$ axis is parallel to the strip.

One can obtain exact results for $\gamma \rightarrow 0$ and $\gamma \gg 1$. The strip width at $\gamma=0$ is $2 w_{0} / \pi$, in accord with the electrostatic sproblem [1]. At $\gamma \gg 1$ the deviation from constant density gradient is small, because electrostatic potential is well screened. In this case, spatial variation of the chemical potential follows that of the density, increasing by $\hbar \omega_{c}$ across the strip. Hence the strip width is $n_{\text {gap }} /|\nabla n|$. 


\section{FIGURES}

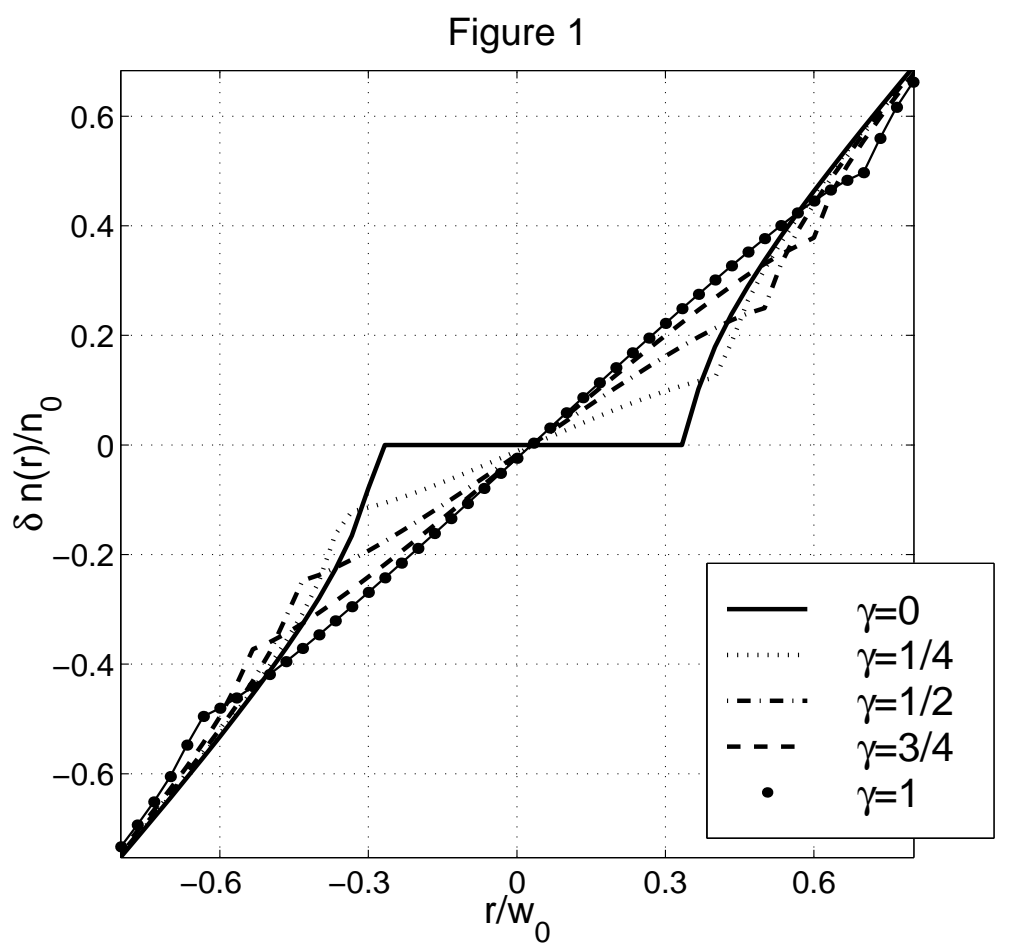

FIG. 1. The effect of localized states on $n(r)$ for 5 values of $\gamma=n_{\text {gap }} / n_{0}$. The units used are $w_{0}=\left((\epsilon+1) \hbar \omega_{c} / 2 e^{2}|\nabla n|\right)^{1 / 2}$ and $n_{0}=\left((\epsilon+1) \hbar \omega_{c}|\nabla n| / 2 e^{2}\right)^{1 / 2}$.

We solve the problem numerically for all $\gamma$ (see Fig.1). In the whole range of $\gamma$ the strip width is reasonably accurately given by the formula

$$
w \approx(2 / \pi+\gamma) w_{0}=(2 / \pi) w_{0}+n_{\text {gap }} /|\nabla n|,
$$

interpolating between the two exactly solvable limits.

A common model for the density of states in a Landau level is a broad line (gaussian or lorentzian) with the states localized in the tail. In this model, the transition between (less compressible) strip and (more compressible) outer region will be more gradual than in the model considered. The estimate (6) for the width of the strip, however, will remain correct, assuming that $n_{\text {gap }}$ measures total number of states in the Landau levels tails.

In the experiment [3], at the $m=2$ plateau, the density of states in the gap $n_{\text {gap }} \approx$ $0.03 n_{\text {total }}$, where $n_{\text {total }}=1.5 \cdot 10^{11} \mathrm{~cm}^{-2}$. The density gradient $\nabla n \approx 2 \cdot 10^{10} \mathrm{~cm}^{-2} / \mu \mathrm{m}$. Substituting this in (㺼), we get $w_{0}=0.3 \mu \mathrm{m}, \gamma \approx 1$. In the fully incompressible case [1], the strip width would be $2 w_{0} / \pi=0.2 \mu \mathrm{m}$. The observed width $0.5 \mu \mathrm{m}$ agrees with Eq.(6) for estimated $\gamma$.

\section{The effect of negative compressibility of the compressible edge}

For a fully incompressible strip $\left(n_{\text {gap }}=0\right)$, the density is constant within it and varies outside as a square root of the distance from the strip edge [1]. Here we study how this behavior is modified due to finite compressibility of the Landau level states. The Thomas-Fermi theory recipe is to use Eq.(四) with $\mu(n)=\delta n / \kappa$, where $\kappa$ is compressibility. Such a model, however, 
is inconsistent, because of the negative sign of $\kappa$ in the QH state [7]. The Thomas-Fermi problem with $\kappa<0$ leads to an unphysical instability.

The difficulty is circumvented by realizing that the exchange interaction in the case of negative compressibility is essentially nonlocal [9]. This motivates using in (11) an effective interaction which is simplest to write in the Fourier representation:

$$
V_{\mathrm{eff}}(k)=\frac{4 \pi e^{2}}{(\epsilon+1)|k|} \Lambda(k), \quad \Lambda(k)>0, \quad \Lambda(0)=1, \quad \Lambda^{\prime}(0)=\frac{(\epsilon+1)}{4 \pi e^{2} \kappa}=-a,
$$

where $a>0$ is the screening length. The interaction (7) with listed restrictions on $\Lambda(k)$ ensures stability as well as correct Hartree interaction and compressibility. Otherwise, one can make a reasonable choice of $\Lambda(k)$ at $k a>1$.

The problem (团) near the strip edge, with $V_{\text {eff }}$ of the form (团) accounting for exchange effects, can be solved by the Wiener-Hopf method [9]. For that, we write $\delta n(x)=n^{+}(x) \theta(x)+n^{-}(x) \theta(-x)$, where $x>0$ is the compressible region, and Fourier transform Eq.(1): $V_{\text {eff }}(k) n_{k}^{+}=-\left(U_{\text {ext }}^{+}(k)+U_{\text {ext }}^{-}(k)\right)$, where $n_{k}^{ \pm}$and $U_{\text {ext }}^{ \pm}(k)$ are analytic in the upper and lower complex $k$ half-planes. The Wiener-Hopf trick requires factoring $V_{\text {eff }}(k)=A_{k}^{+} / A_{k}^{-}$, where \pm indicates the analyticity half-plane. Then, $A_{k}^{-} U_{\text {ext }}^{+}(k)=$ $\left[A_{k}^{-} U_{\text {ext }}^{+}(k)\right]^{+}+\left[A_{k}^{-} U_{\text {ext }}^{+}(k)\right]^{-}$which yields $n_{k}^{+}=-\left[A_{k}^{-} U_{\text {ext }}^{+}(k)\right]^{+} / A_{k}^{+}$.

We use $V_{\text {eff }}(k)$ of the form (7) with

$$
\Lambda(k)=\exp \left[-a k\left(1-(2 / \pi) \tan ^{-1}(k / \lambda)\right)\right],
$$

and obtain a Wiener-Hopf solution in a closed form. Here the parameter $\lambda$ regularizes the interaction at large $k$ (and small $r$ ): $V_{\text {eff }}\left(r \ll \lambda^{-1}\right)=e^{-2 \lambda a / \pi} V(r)$. Factoring this $V_{\text {eff }}(k)$ gives

$$
A_{k}^{+}=\frac{4 \pi e^{2}}{(k-i \delta)^{1 / 2}}\left(\frac{\delta+i k}{\lambda+i k}\right)^{i a k / \pi} \quad, \quad A_{k}^{-}=(k+i \delta)^{1 / 2}\left(\frac{\delta-i k}{\lambda-i k}\right)^{i a k / \pi}
$$

where $\delta=+0$. Near the edge $U_{\text {ext }}(x)=E x+c$, and thus

$$
n_{k}^{+}=\frac{(\epsilon+1) E}{4 \pi e^{2}} \frac{\left(i \delta^{*}\right)^{1 / 2}}{(k-i \delta)^{3 / 2}}\left(\frac{k-i \lambda}{k-i \delta}\right)^{i a k / \pi}
$$

where $\delta^{*} \sim w_{0}^{-1}$. The inverse Fourier transform of $n_{k}^{+}$gives the charge distribution near the strip edge. Note the asymptotic behavior of $\delta n(x): \delta n\left(x \gg \lambda^{-1}\right)=2 n_{0}\left(x / \pi w_{0}\right)^{1 / 2}$, $\delta n\left(x \ll \lambda^{-1}\right)=2 n_{0}\left(x / \pi w_{0}\right)^{1 / 2} e^{\lambda a / \pi}$. Here we expressed $E$ and $\delta^{*}$ in terms of $w_{0}$ and $n_{0}$.

The solution shows that at large screening length $a$ there is a significant departure of the density near the edge from the square root profile of [1]. The density profile becomes nonmonotonic at $a \lambda \gg 1$. The interpretation of this result is that as the density is lowered, electrons at the edge form a one dimensional Wigner solid at densities such that the interior of the system is still fluid.

We studied numerically the effect of exchange on the strip width. As the exchange interaction parameter increases, the strip becomes wider (see Fig.21). In the simulation, a model interaction 


$$
V_{\text {eff }}(r)=\alpha /|r|+(1-\alpha) /\left(r^{2}+\tilde{a}^{2}\right)^{1 / 2}
$$

was used, with $\tilde{a}=a /(1-\alpha)$. Similar to the Wiener-Hopf solution for an isolated edge, at large values of the exchange parameter the density profile becomes nonmonotonic. Note that our density functional, being quadratic in $n(r)$, obeys an exact particle-hole symmetry. Hence the density profiles on the upper and lower sides of the plateau in Fig.2 are identical up to a sign change.

Figure 2

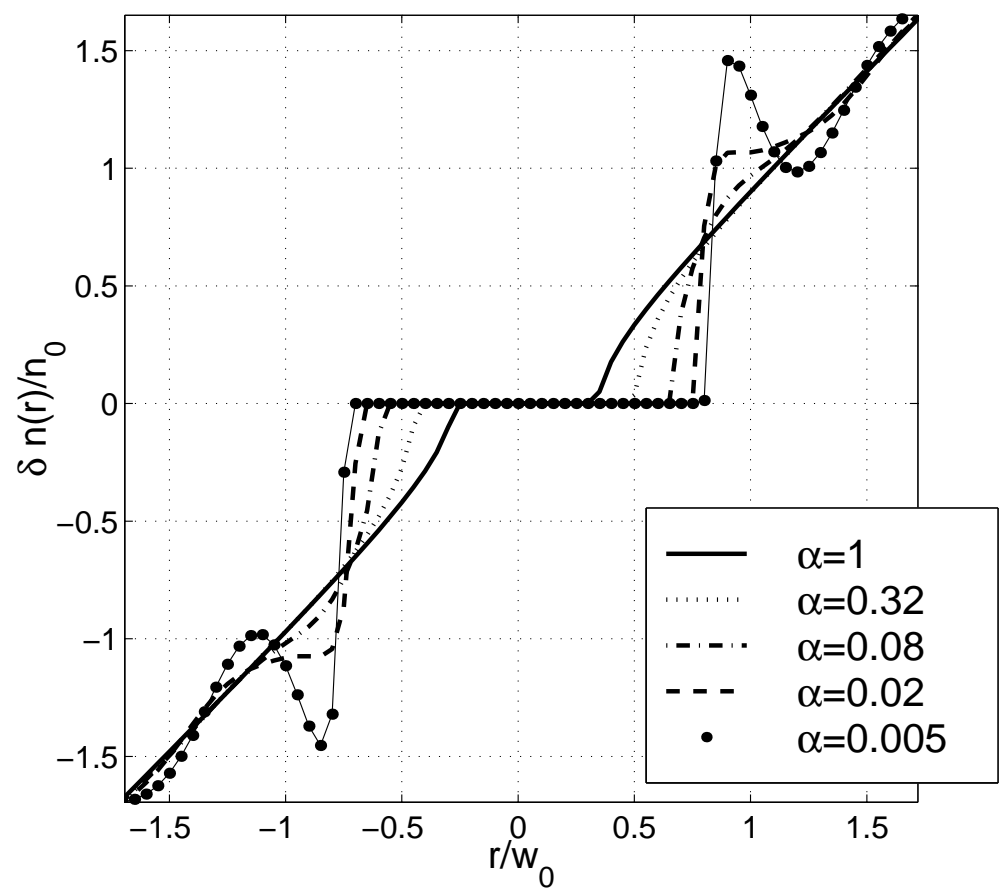

FIG. 2. Incompressible strip density distribution created by effective interaction (11) shown for 5 values of the exchange parameter $\alpha$, with $\tilde{a}=0.5 w_{0}$.

In conclusion, we find that finite density of localized states and electron exchange interaction both have similar effect on the width of the incompressible strip. The strip width increases as a function of the localized states density, and as a function of electron exchange parameter. However, the density profile in these two problems evolves differently. For a high density of localized states the density gradient becomes nearly uniform, whereas at large exchange the plateau in the density distribution becomes wider. At very large exchange, the density profile becomes nonmonotonic, indicating formation of a one dimensional Wigner crystal at the edge.

L.L. is grateful to R. Ashoori, G. Finkelstein, T. D. Fulton, and A. Yacoby for useful discussions of their data. Research at MIT is supported in part by the MRSEC Program of NSF under award 6743000 IRG.

\section{References}

1. D. B. Chklovskii, B. I. Shklovskii, and L. I. Glazman, Phys. Rev. B 46, 4026 (1992).

2. S. H. Tessmer, P. I. Glicofridis, R. C. Ashoori, L. S. Levitov, M. R. Melloch, Nature, 392, No.6671, 51 (1998); 
G. Finkelstein, P. I. Glicofridis, S. H. Tessmer, R. C. Ashoori, M. R. Melloch, preprint 3. A. Yacoby, H. F. Hess, T.A. Fulton, L. N. Pfeiffer and K. W. West, Solid State Communications, 111, 1-13 (1999)

4. K. L. McCormick, M. T. Woodside, M. Huang, M. S. Wu, P. L. McEuen, C. Duruoz, J. S. Harris, Phys. Rev. B, 59, 4654 (1999)

5. Y. Y. Wei, J. Weis, K. v. Klitzing, K. Eberl, Phys. Rev. Lett., 81, 1674 (1999)

6. I. A. Larkin, J. H. Davies, Phys. Rev. B, 52, R5535 (1995)

7. J. P. Eisenstein, L. N. Pfeiffer, and K. W. West, Phys. Rev. Lett. 68, 674 (1992);

B. Tanatar, D. M. Ceperley, Phys. Rev. B 39, 5005 (1989)

8. A. L. Efros, cond-mat/9905368

9. I. A. Larkin, L. S. Levitov, to be published 